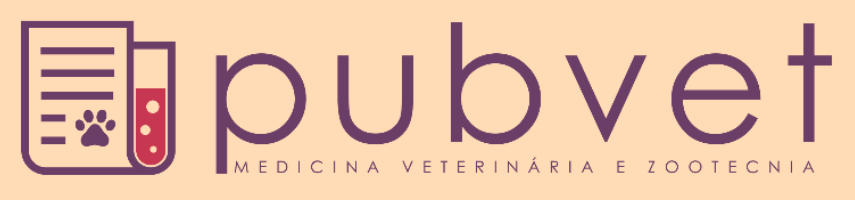

https://doi.org/10.31533/pubvet.v15n01a736.1-11

\title{
Aspectos reprodutivos e resolução cirúrgica da distocia em primatas não-humano
}

\author{
Jacqueline Souza Santana ${ }^{10 *}$, Murilo Neves Borges de Oliveira ${ }^{2} \bullet$, Bruna Ros Soares $^{30}$, Mario \\ Roberto Vianna da Silva ${ }^{3}$, Délcio Almeida Magalhães ${ }^{30}$, Fabiana Sperb Volkweis ${ }^{30}$ \\ ${ }^{1}$ Professora Me. Doutoranda (Unesp) de Medicina Veterinária, Centro Universitário do Planalto Central Apparecido dos Santos. Gama-DF. Brasil. \\ ${ }^{2}$ Graduando em Medicina Veterinária, Centro Universitário do Planalto Central Apparecido dos Santos (UNICEPLAC). Gama-DF. Brasil \\ ${ }^{3}$ Graduando em Medicina Veterinária, Universidade Anhembi Morumbi (UAM). São Paulo - SP. Brasil. \\ *Autor para correspondência: E-mail: jacquesoouza@gmail.com
}

\begin{abstract}
Resumo. Os primatas não-humanos, bem como as demais espécies selvagens, demandam que mais pesquisas sejam desenvolvidas com o objetivo de proporcionar o conhecimento da sua biologia reprodutiva e métodos de manejo aplicados à reprodução, dentre elas a cesariana é critério de eleição em alguns casos de distocia, onde há dificuldade no momento do parto por origem materna ou fetal. A pelvimetria radiográfica vem sendo empregada como método de diagnóstico nesses casos, além da ultrassonografia. Portanto, o manejo reprodutivo como a cesárea, pode auxiliar na diminuição da taxa de mortalidade, contribuindo também na preservação e outras técnicas que distribuem sua diversidade genética, assim, reduzindo o número de espécies ameaçadas, mantendo uma população biologicamente viável.
\end{abstract}

Palavras-chave: Cesariana, distocia, pelvimetria, primatologia

\section{Reproductive aspects and surgical resolution of dystocia in non- human primates}

\begin{abstract}
Non-human primates, as well as other wild species, demand that more research be carried out in order to provide knowledge of their reproductive biology and management methods applied to reproduction, including cesarean section is the criterion of choice in some cases. dystocia, where there is difficulty at the time of delivery due to maternal or fetal origin. Radiographic pelvimetry has been used as a diagnostic method in these cases, in addition to ultrasound. Therefore, reproductive management such as cesarean section can help reduce the mortality rate, also contributing to preservation and other techniques that distribute their genetic diversity, thus reducing the number of threatened species, maintaining a biologically viable population.
\end{abstract}

Keywords: Caesarean, dystocia, pelvimetry, primatology

\section{Aspectos reproductivos y resolución quirúrgica de la distocia en primates no humanos}

Resumen. Los primates no humanos, así como otras especies silvestres, exigen el desarrollo de más investigaciones con el fin de brindar conocimiento de su biología reproductiva y métodos de manejo aplicados a la reproducción, entre ellos la cesárea es el criterio de elección en algunos casos de distocia, donde hay dificultad en el parto debido al origen materno o fetal. La pelvimetría radiográfica se ha utilizado como método diagnóstico en estos casos, además de la ecografía. Por tanto, el manejo reproductivo, como 
la cesárea, puede ayudar a disminuir la tasa de mortalidad, contribuyendo también a la conservación y otras técnicas que distribuyen su diversidad genética, reduciendo así el número de especies amenazadas, manteniendo una población biológicamente viable.

Palabras clave: Cesárea, distocia, pelvimetría, primatología

\section{Introdução}

As pesquisas científicas em primatas não-humanos $(\mathrm{PNH})$ vêm progredindo devido a sua importância na conservação das espécies e um conhecimento mais aprofundado com relação à fisiologia, nutrição e anatomia dessas espécies. As espécies são subdivididas em duas infra-ordens, primatas do velho mundo pertencentes à ordem Catarhini e do novo mundo como os Platyrrhini (Presgrave, 2002).

O Brasil possui uma variedade de espécies de animais, em que alguns se encontram extintos, incluindo os PNH. É perceptível que a caça, a destruição de habitats e até mesmo o comércio ilegal são os dos motivos desse aumento na lista de animais ameaçados de extinção (Bressan et al., 2009; Ferrari \& Rímoli, 1985).

Os zoológicos, além da área de lazer e expositora de animais, expandiram sua finalidade em pesquisas na reprodução das espécies ameaçadas e na educação ambiental, com isso, colabora para a preservação de habitats e espécies em risco de extinção (Garcia \& Bernal, 2015).

$\mathrm{O}$ manejo reprodutivo em PNH apresenta uma metodologia para melhorar o parto e nascimento dos filhotes, utilizando a intervenção cirúrgica em casos de distocias, assim diminuindo o risco de natimortos e abortos evitando a extinção dos mesmos. Essa técnica é indicada por mau posicionamento ou desenvolvimento fetal, tamanho aumentado dos fetos, redução do canal pélvico da fêmea, inércia uterina ou putrefação fetal (Fossum, 2014). A cesariana conhecida também como histerotomia, é considerada um procedimento para o tratamento de distocia, sendo a causa mais frequente, anormalidades que comprometam o nascimento dos filhotes. Esse método deve ser feito de forma segura para a cria e progenitora, principalmente quando realizada o mais próximo do início do trabalho de parto (Simas et al., 2012).

Este trabalho tem como objetivo intensificar a importância da conservação dessas espécies, utilizando o manejo reprodutivo como forma de garantir maior número de nascimentos de PHN.

\section{Primatas do novo e velho mundo e a importância da conservação dessas espécies}

A ordem dos primatas corresponde a um grupo de mamíferos com classificações cuja suas denominações estão relacionadas com a taxonomia da espécie. Existem muitas polêmicas referente à classificação dos PNH, atualmente é subdividida em Catarrhini (primatas do velho mundo), tendo como exemplo o Pongo abelli, e os Platyrrhini (primatas do novo mundo) como os Alouatta spp. (Fortes \& Bicca-Marques, 2005).

Os primatas encontrados no continente americano se originaram da América do Norte e migraram para América do Sul, em seguida para África. As espécies distinguem-se pela posição das aberturas nasais e a presença ou ausência de cauda preênsil, onde orifícios voltados para baixo e sem cauda, são do velho mundo, e quando as possui, não é preênsil. Primatas do novo mundo na maioria das espécies consideram-se de pequeno porte com cauda preênsil (Presgrave, 2002).

O Brasil é considerado um depositário da maior distinção de primatas no mundo tendo cerca de 118 espécies de PNH. A falta de conhecimento sobre a importância destes animais na cadeia trófica, na ciclagem de nutrientes, dispersão de sementes e na polinização, contribui para uma falha no entendimento na compreensão da preservação dessas espécies. As maiores causas de extinção são o desmatamento, a caça indiscriminada e destruição dos habitats (Couto-Santos et al., 2004). O aumento na ocorrência da destruição de habitats naturais põe a sobrevivência dos primatas em risco em diversos locais. Alguns animais da fauna brasileira compõem a lista de espécies ameaçadas no Brasil, dentre os primatas $35 \mathrm{sp}$. na qual 14 são vulneráveis (VU), 15 em perigo (EN) e 6 criticamente em perigo (CR) (Machado et al., 2008). 
Mesmo com conservacionistas e criação de áreas protegidas, os primatas e outras espécies de mamíferos ainda enfrentam a fragmentação de habitat, pecuária, agricultura, apanha, desmatamento, expansão urbana, vulnerabilidade a epidemias, hibridação com espécies invasoras e a caça ilegal, na qual sofre com impactos negativos de intervenções antropogênicas (Machado et al., 2008; Vidal et al., 2012). Como forma preventiva de reduzir a lista de espécies ameaçadas, o melhoramento no manejo reprodutivo e preservação de genes, auxilia nessa redução. Por falta de pesquisa, as técnicas estudadas são provenientes de animais domésticos sendo adaptadas aos animais silvestres (Teixeira, 2005).

Para Ferrari \& Rímoli (1985) é interessante selecionar os animais com melhores características para reprodução, aumentando sua população em cativeiro e em vida livre a longo prazo. Partindo de uma visão científica, há uma continuidade na natureza, onde compreendendo os processos comportamentais em animais, contribui para compreensão de organismos mais complexos como o homem (Auricchio, $\underline{1995)}$.

\section{Aspectos anatômicos}

A estrutura do aparelho reprodutor feminino dos primatas é constituída pelo útero, tubas uterinas, ovários, vagina e vulva. Na qual a espécie humana, possui os grandes e pequenos lábios, vestíbulo e o clítoris externos. Já nos demais primatas os grandes lábios externos são ausentes, e o vestíbulo é interno que comunica a vagina com a vulva (Souza, 2010).

O útero se encontra na pelve entre a vesícula urinária e dorsalmente ao intestino delgado, formados por um corpo, cornos uterinos e cérvix, como mostra a figura 1 (Dyce et al., 2004).

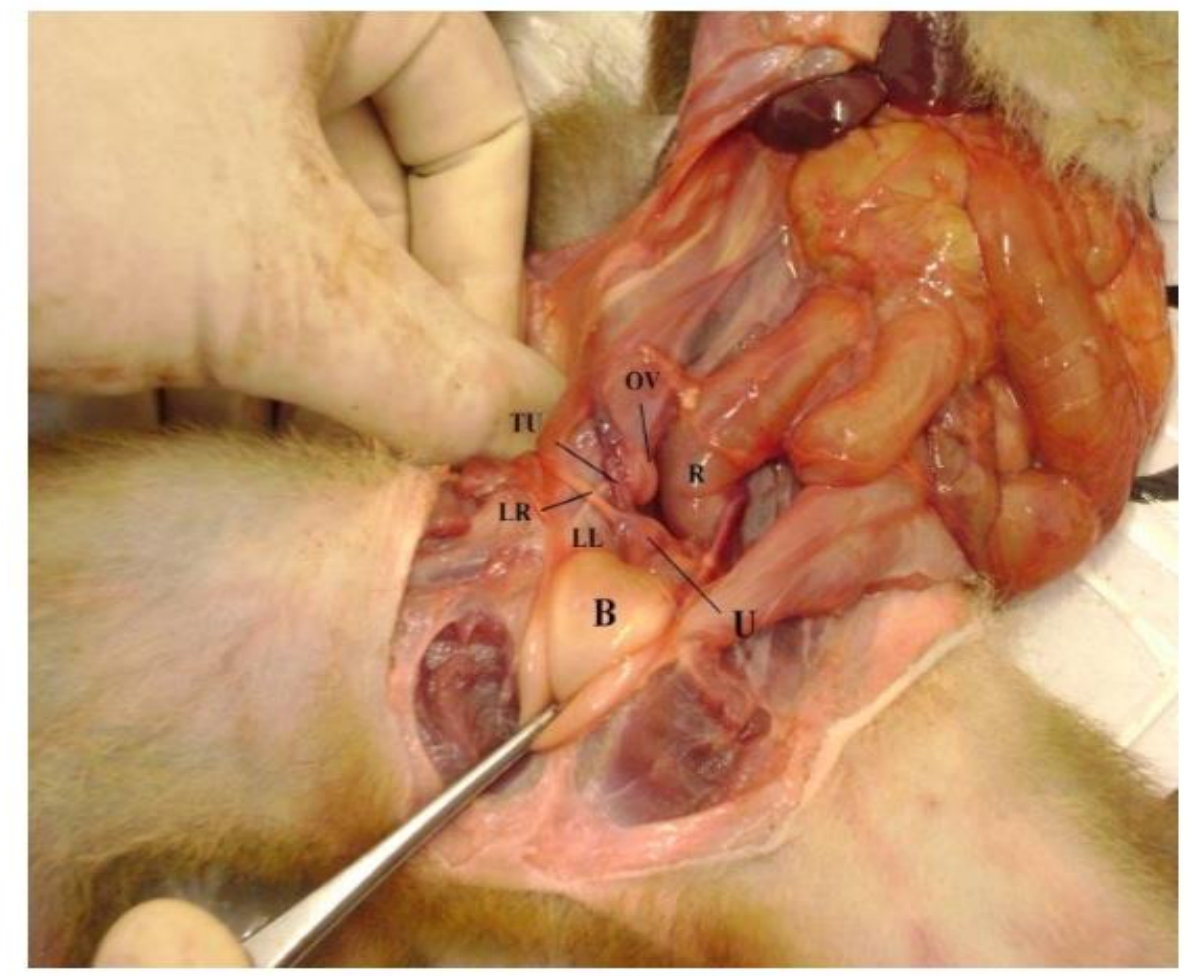

Figura 1. Fotografia de órgão reprodutor feminino intra-abdominal de PNH. Região Pélvica B - Vesícula Urinária; LL - Ligamento largo; LR - Ligamento redondo; OV Ovário; R - Reto; TU - Tuba uterina; U - Útero. Fonte: Souza (2010).

A parede do útero é dividida em três áreas, endométrio, miométrio e perimétrio, em que é formado por uma túnica serosa e sub serosa proveniente do peritônio, vista como uma porção espessada no fundo do útero e rica em fibras elásticas (Souza, 2010).

O miométrio é constituído de tecido muscular liso, que regula a hemodinâmica vascular e a função mecânica para manter e realizar a expulsão do feto. Já o endométrio é formado por um epitélio espessado que possuem glândulas que se ramificam próximas ao miométrio. A cérvix formada por epitélios que 
secretam muco tem grande importância para o manejo reprodutivo, pois se houver falhas podem resultar em baixa fertilidade e esterilidade (Souza, 2010).

O útero em primatas primitivos como os Plesiadapis era composto por dois cornos uterinos (útero bicornual) já os da ordem Catarrhini e Platyrrhini apresentam um corpo uterino simples, como na figura $\underline{2}$ (Souza, 2010). O tamanho do útero diversifica de acordo com o tipo de espécie, em que o corpo uterino é revestido lateralmente pelo ligamento largo, unindo-se com o peritônio na cérvix (Moore, 2013).

As tubas uterinas conduzem para cavidade uterina os oócitos liberados através dos ovários, visto que é a porção onde ocorre a fertilização. São divididas em quatro partes, infundíbulo, ampola, istmo e parte uterina, onde a fração terminal da tuba atravessa a parede do útero pelo o óstio uterino (Moore, 2013).

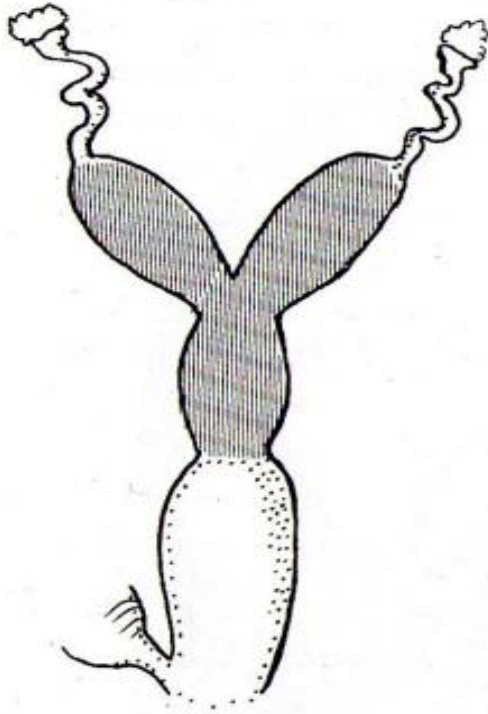

A

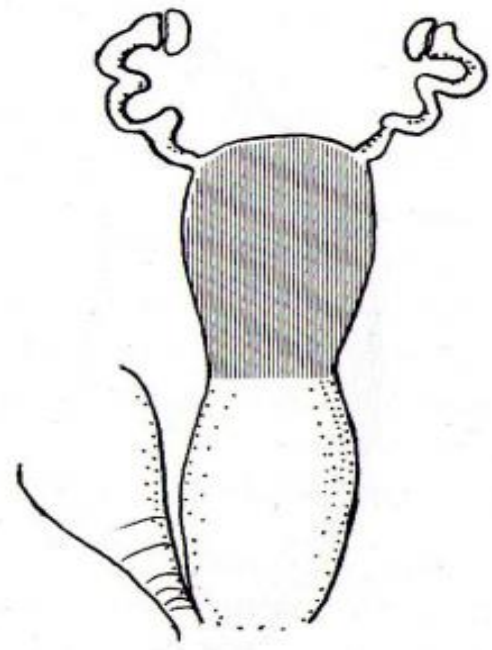

B

Figura 2. Imagem ilustrativa de órgão reprodutor feminino de PNH. Útero bicornual dos primatas prossímios (A), útero simples dos primatas antropóides (B). Fonte: Souza (2010).

Os ovários por sua vez, possuem funcionalidade endócrina e gametogênica. Proximal ao corno uterino encontra-se suspenso pela parte cranial do ovário denominada mesovário, na qual cria uma bolsa para reter os mesmos. A vagina é uma passagem reprodutiva que vai da cérvix até a entrada da uretra, já sua porção caudal é o vestíbulo, onde se encontra desde o orifício uretral até a vulva externa (Dyce et al., 2004).

Em alguns Platyrrhini o clitóris anterior há ambos os lábios, são largos e pendurados podendo facilmente ser confundido com um órgão reprodutor masculino, o pênis; enquanto em outros primatas, esse segmento é pequeno e encapuzado (Wolfe-Coote, 2005).

Nas espécies de primatas fêmeas, uma das formas de diferenciá-las é a da placenta, na qual as membranas placentárias se espalham por toda cavidade uterina, podendo sua circulação fetal ser separada da materna por várias camadas de tecido, como a epiteliocorial. Já na hemocorial encontra-se localizada em um ou dois discos discretos, onde há uma aproximação maior entre os suprimentos sanguíneos fetais e maternos (Fleagle, 2013).

De acordo com Landim-Alvarenga et al. (2006) a placenta é um órgão intermediário entre a mãe e o feto que serve para suprimento de oxigênio e nutrientes, remoção de detritos metabólicos, produção e secreção de hormônios, fatores de crescimento fetal e regulação do ambiente uterino do feto.

$\mathrm{Na}$ tabela 1 podemos observar dados sobre tipos de útero e placenta de acordo com a espécie citada: tempo de gestação (dias), tamanho da ninhada/massa média de cada neonato, idade ao desmame (dias), idade da primeira reprodução feminina (anos) (Wolfe-Coote, 2005). 
Tabela 1. Parâmetros reprodutivos de fêmeas primatas

\begin{tabular}{|c|c|c|c|c|c|}
\hline Taxonomia & $\begin{array}{c}\text { Útero (Bicornual ou } \\
\text { Simples)/Placenta } \\
\text { (Epiteliocorial ou } \\
\text { Hemocorial }\end{array}$ & $\begin{array}{c}\text { Duração da gestação } \\
\text { (Dias) }\end{array}$ & $\begin{array}{c}\text { Tamanho da } \\
\text { Ninhada/massa } \\
\text { média por neonato } \\
(\mathrm{g})\end{array}$ & $\begin{array}{c}\text { Idade ao desmame } \\
\text { (dias) }\end{array}$ & $\begin{array}{l}\text { Idade da primeira } \\
\text { reprodução } \\
\text { feminina (anos) }\end{array}$ \\
\hline Microcebus murino & $\mathrm{B} / \mathrm{E}$ & 60 & $2 / 4.6$ & 40 & 1 \\
\hline Lemur catta & $\mathrm{B} / \mathrm{E}$ & 135 & $1 / 85$ & 179 & 2 \\
\hline Loris tardigradus & $\mathrm{B} / \mathrm{E}$ & 166 & $1 / 10$ & 170 & 1.5 \\
\hline Nycticebys coucang & $\mathrm{B} / \mathrm{E}$ & 193 & $1 / 50.8$ & 180 & 2.1 \\
\hline Galago senegalensis & $\mathrm{B} / \mathrm{E}$ & 142 & $1 / 19$ & 98 & 1.4 \\
\hline Otolemur Crassicaudatus & $\mathrm{B} / \mathrm{E}$ & 135 & $2 / 43.2$ & 135 & 2.2 \\
\hline Saimiri sciureus & $\mathrm{S} / \mathrm{H}$ & 170 & $1 / 106.4$ & 168 & 2.5 \\
\hline Aotus trivirgatus & $\mathrm{S} / \mathrm{H}$ & 133 & $1 / 94$ & 75 & 2.4 \\
\hline Callitrix jacchus & $\mathrm{S} / \mathrm{H}$ & 148 & $2 / 27$ & 60 & 1.4 \\
\hline Saguínus oedipus & $\mathrm{S} / \mathrm{H}$ & 168 & $2 / 42,1$ & 50 & 1.9 \\
\hline Chlorocebus aethiops & $\mathrm{S} / \mathrm{H}$ & 163 & $1 / 335.9$ & 201 & 5 \\
\hline Papio anubis & $\mathrm{S} / \mathrm{H}$ & 180 & $1 / 915$ & 584 & 4.5 \\
\hline Macaca mulatta & $\mathrm{S} / \mathrm{H}$ & 165 & $1 / 466.3$ & 192 & 3 \\
\hline Macaca fascicularis & $\mathrm{S} / \mathrm{H}$ & 160 & $1 / 326.1$ & 330 & 3.9 \\
\hline Homo (Pan) troglodytes & $\mathrm{S} / \mathrm{H}$ & 235 & $1 / 1.750$ & 1680 & 13 \\
\hline Homo sapiens & $\mathrm{S} / \mathrm{H}$ & 267 & $1 / 2.900$ & 730 & 14 \\
\hline
\end{tabular}

Fonte: Adaptado: Wolfe-Coote (2005).

\section{Fisiologia da cópula e gestação}

Pesquisas relacionadas à reprodução, contribuem como uma metodologia aplicada em programas de conservação de espécies ameaçadas, com ou sem auxílio de técnicas de reprodução assistida, como fertilização in vitro (Araújo, 2012). De acordo com Presgrave (2002), o acasalamento varia de acordo com as espécies, já que existem monogâmicos, poligâmicos e promíscuos. Ocorre de forma sazonal associado aos fatores ambientais, tais como: fotoperíodo, temperatura, umidade e disponibilidade de alimento.

A fase reprodutiva inicia-se com a puberdade na qual altera os hormônios sexuais. Os Catarrhini são detectados com a primeira menstruação, já os Platyrrhini pelo aumento do peso corporal. Algumas espécies apresentam o sex skin, manifestada como vermelhidão e inchaços na parte genital tanto dos machos, quanto das fêmeas (Presgrave, 2002).

Nas diversas espécies de primatas fêmeas, a fase de sexualidade pode ser dividida em três segmentos, como atratividade, proceptividade e receptividade. Na atração é onde ocorre o encanto sexual do macho pela fêmea através de características na mudança de coloração da pelagem, odor e temperatura. A proceptividade é manifestada por meio de vocalização e toque. Já a receptividade demonstra a fase da aceitação da copulação e ejaculação do macho (Rodrigues, 2010).

Após a concepção, há preocupações durante a gestação e no pós-parto. Há algumas afecções durante a gravidez como, sangramento vaginal no qual é considerado normal durante o 1-50 dias de gestação, sendo mais comum do dia 18-45, entretanto não é vista em todas as espécies. Já o descolamento de placenta é avaliado um fator de emergência visto que pode resultar em hemorragias, geralmente encontradas na cavidade uterina e não é visualizada externamente (Courtney, 2012).

O aborto geralmente ocorre no início da prenhez e não é detectado. O mecanismo exato do aborto é incerto em muitos casos, tanto em humanos como em animais. Alguns podem ser devidos a um equilíbrio hormonal materno abaixo do ideal comprometendo a continuidade da gravidez, enquanto outros provavelmente estão relacionados a fatores tais como traumas, neoplasia uterina, defeitos anatômicos, condições infecciosas sendo elas, Listeria, Salmonella, Pneumococccus, Toxoplasma, Adenovírus (Cooper \& Hull, 2017; Courtney, 2012).

Nas diversas espécies o equilíbrio entre diferentes hormônios é essencial pela continuidade da gestação, dentre elas a progesterona, estrógeno, relaxina e prolactina. Para o trabalho de parto é utilizado progesterona, estrógeno e ocitocina. Entretanto, a diminuição de progesterona/estrógeno é o acontecimento de maior relevância na gestação, pois sucede a dilatação da cérvix e no aumento da contratilidade uterina (Veiga et al., 2009). 
A exigência de energia e nutrientes aumenta durante a gestação e lactação. Visto que o feto depende das reservas maternas, e o neonato da produção láctea da mãe. A progesterona exerce na preservação dessa energia, e o estrógeno responsável pelo encaminhamento da energia para o feto, à vista disso a importância do peso da fêmea gestante (Prestes \& Landim-Alvarenga, 2017).

O tempo de gestação varia de acordo com as espécies, como mostrado anteriormente. Embora existam intervalos considerados normais, cada grupo pode ter uma data gestacional média que difere de outro grupo de animais, permanecendo dentro dos intervalos publicados (Courtney, 2012).

\section{Distocia em primatas não-humanos}

De acordo com o progresso da gestação esta pode ser classificada como normal ou distócica, quando acontece de forma fisiológica ou à medida que necessita de intervenção manipulativa, medicamentosa ou cirúrgica (Nelson \& Couto, 2015). A distocia é definida no momento em que no parto e expulsão do feto, advém de uma anormalidade ou dificuldade, seja de origem materna ou fetal. Podendo ser disfunção uterina, contrações insuficientes ou descoordenadas, mau posicionamento fetal, mau desenvolvimento fetal, desproporção do tamanho do feto com o canal pélvico, ou anormalidade anatômicas da pelve resultando como um obstáculo no canal do parto (Stockinger et al., 2011).

Além da má representação, outras causas de distocia documentadas em PNH incluem fetos macrossômicos, nascimento de múltiplos fetos, massas uterinas, deficiências nutricionais e fraturas traumáticas dos ossos. Macacos-esquilo, por exemplo, são um modelo para distocia devido a fetos macrossômicos (Stockinger et al., 2011). Distocia em macacos pode ser um problema sério e levar a morbidade infantil e materna, portanto os fatores de risco para natimortos, que podem resultar de distocia foram estabelecidos para muitas espécies de PNH. Nos chimpanzés, o aumento da paridade e idade tem sido associado com fetos aumentados. Em babuínos, a idade materna, e o peso foi associado à perda fetal. Nos macacos rhesus, a idade materna tem sido associada ao aumento fetal (Stockinger et al., 2011).

Um dos tratamentos medicamentosos é via fármacos para indução da contração uterina, quando há uma atonia uterina, na qual a ocitocina é a mais empregada. Associada a ela, pode-se utilizar o gluconato de cálcio. Nas situações de distocia em que o tratamento medicamentoso não foi eficaz, ou não poderá ser realizado, o procedimento de escolha é a cesariana (Lúcio, 2008).

De acordo com Stockinger et al. (2011), as indicações para praticar cesariana são: Inércia uterina parcial primária ou secundária sem resposta ao tratamento médico, tamanho fetal excessivo em relação ao canal do parto, má formações do canal pélvico, apresentação anormal do feto sem possibilidade de manipulá-lo, putrefação fetal, doença sistêmica da mãe, suspeita de torção uterina, ruptura, prolapso ou hérnia e evidência de sofrimento fetal.

A proporção elevada de cesarianas anteriores são um determinante significativo da distocia, podendo ser prejudicial. Portanto, a idade gestacional e o total de nascimentos anteriores também contribuíram para o risco de parto distócico. A Universidade de Washington limitou para três, o número de cesarianas realizadas em um macaco, incluindo cirurgias necessárias experimentalmente e clinicamente. É viável aplicar a interação da proporção de nascidos vivos em comparação com a proporção de intervenções cirúrgicas, para determinar o risco geral de um animal e dedicar recursos conforme o necessário (Stockinger et al., 2011).

\section{Diagnóstico da distocia}

O diagnóstico da distocia consiste em uma boa anamnese, auxiliada pelo histórico do paciente como antecedentes reprodutivos, exame físico, exames complementares, palpação abdominal, seguida da radiografia para quantificar caso seja confirmatória a gravidez e a ultrassonografia para visualizar a viabilidade dos fetos, tamanho e posição (Bojrab, 2005; Fossum, 2014; Niebauer, 1993). As causas do diagnóstico clínico incluem mudanças comportamentais sutis, como inquietação, alimentação alterada, micção frequente, manipulação digital da genitália, placenta prévia, ruptura uterina, descolamento da placenta, fraqueza materna e trabalho de parto prolongado. Na qual, o trabalho de parto normal em primatas não humanos ocorre durante 5 a 7 horas (Stockinger et al., 2011). 
Além da radiografia convencional, à pelvimetria radiográfica na qual é a determinação métrica das dimensões pélvicas, vem sendo utilizada como forma profilática de complicações que podem acontecer durante o parto, permitindo também, a classificação anatômica e obstétrica da pelve. Sendo de baixo custo e fácil acesso, pode ser empregada para determinar se a fêmea é apta ou não para reprodução (Pafaro et al., 2010).

Para efetuar as radiografias da pelve, o paciente se encontra em decúbito dorsal para efetuar uma projeção ventro-dorsal para mensuração do diâmetro biilíaco superior (DBIS); diâmetro biíliaco inferior (DBII); diâmetro biilíaco médio (DBIM); diâmetro diagonal direito (DDD); diâmetro diagonal esquerdo (DDE); diâmetro sacropúbico (DSP) e área de entrada da pelve (AEP) de acordo com a figura 3 abaixo (Valle et al., 2006).

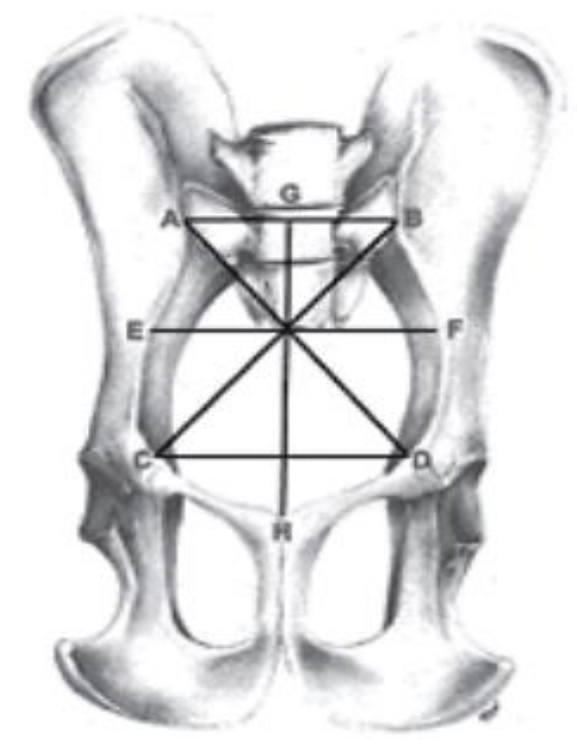

Figura 3. Representação esquemática da mensuração dos diâmetros da pelve de um Aotus azarai infulatus. Fonte: Valle et al. (2006).

\section{Tratamento}

A cesariana ou histerotomia baseia-se na abertura do útero para remoção dos fetos, é indicada quando as intervenções obstétricas não são eficazes. Podendo ser utilizada de forma emergencial em casos de afecções maternas e fetais, resultando em distocia ou eletiva em algumas espécies específicas (Bojrab, 2005; Fossum, 2014; Niebauer, 1993).

Para dar início ao procedimento cirúrgico, os requisitos de jejum devem levar em consideração o tamanho, a idade e o estado de saúde do animal, bem como a hora do dia em que o procedimento ocorrerá e o anestésico a ser utilizado. Para a maioria dos primatas não humanos, o jejum alimentar indicado é de no mínimo 4 horas podendo ser estendido até 8 horas para reduzir o risco de vômito e aspiração. Já o jejum hídrico, não deve ser realizado(Rowsell, 1991).

Os protocolos anestésicos utilizados em fêmeas gestantes devem ser realizados de forma segura para a mãe e o feto, e ao mesmo tempo empregar a terapia antálgica adequada para a realização da cirurgia (Lavor et al., 2004). Levando em consideração alterações que podem ocorrer na perfusão uterina, fornecimento de oxigênio para o feto e uma possível depressão cardiorrespiratória dos neonatos após o parto (Fantoni \& Cortopassi, 2009).

Como medicação pré-anestésica, a associação tiletamina/zolazepam, é muito utilizada para anestesia de procedimentos não dolorosos ou contenção química em várias espécies de animais selvagens. A utilização de anestésico inalatório também pode ser empregada de acordo com o tamanho da espécie (Klaumann \& Otero, 2013). Os benzodiazepínicos podem causar uma depressão cardiorrespiratória fetal, letargia, apnéia e hipotermia nos recém-nascidos, porém essas alterações possam ser evitadas com a redução das doses e monitoração adequada da paciente desde o pré-operatório (Fantoni \& Cortopassi, 2009). 
A indução anestésica pode ser realizada com propofol (Williams et al., 2014). A preparação e os procedimentos para intubação após a indução devem ser determinados previamente com base no animal em particular. O uso do tubo endotraqueal é recomendado para manter as vias aéreas abertas (Rowsell, 1991) no entanto a intubação pode ser difícil em algumas espécies menores (Williams et al., 2014). A anestesia pode ser mantida com isofluorano (CAM de 0,5 a 2,5\%) vaporizado em $100 \%$ de oxigênio (Prestes et al., 2014). Atenção deve ser tomada quando da administração de anestésicos inalatórios, pois, quanto menor seu peso molecular, maior a passagem transplacentária o que pode provocar o feto, por isso, torna-se fundamental manter um plano adequado para o procedimento cirúrgico, evitando-se níveis profundos para que não ultrapasse a barreira placentária (Lavor et al., 2004).

Os analgésicos devem ser administrados antes da cirurgia permitindo tempo suficiente para atingir o efeito máximo antes dos estímulos cirúrgicos, e após o procedimento sendo fornecidos conforme necessário para manter um nível estável de conforto para o animal (Rowsell, 1991), podendo ser utilizada inicialmente a combinação de Meloxicam e Tramadol, pois a combinação de opióides e AINEs é muito utilizada, para terapia de dores agudas e moderadas (Prestes et al., 2014).

Para iniciar a intervenção cirúrgica é necessário colocar a fêmea gestante em decúbito dorsal, realizar remoção de pelos do local e em seguida a antissepsia com iodo povidine e álcool (Prestes et al., 2014). Inicia-se a intervenção cirúrgica com uma incisão na linha média ventral do umbigo até a porção proximal do púbis, logo após encontrar musculatura, efetue-se uma incisão punctória na linha alba para acessar cavidade abdominal (Mosdow, 1976). Ao localizar o útero gravídico, o mesmo deve ser isolado com compressas estéreis e a incisão feita em uma região menos vascularizada na face ventral ou dorsal do corpo uterino, de extensão suficiente para permitir a passagem dos fetos, na qual cada neonato será relocado para o local da incisão (Fossum, 2014). Em seguida, romper o saco amniótico de cada neonato e pinçar o cordão umbilical evitando contaminação do leito cirúrgico e abdômen. A placenta é expelida junto com os fetos, porém se esta não tiver se separado, deve ser removida do endométrio cuidadosamente para não ocorrer hemorragia (Fossum, 2014).

Após a remoção de todos os fetos, inicia-se a sutura da incisão uterina com fio absorvível poliglecaprone 25 , com um padrão simples contínuo na primeira camada, em seguida um padrão invaginante de Cushing, com fio de mesma característica. O peritônio e a musculatura abdominal podem ser suturados com vicryl/poliglactina em um padrão simples contínuo, a obliteração do espaço morto feito no padrão zigue e zague com o fio citado acima, e a dermorrafia realizada em padrão sultan com fio não absorvível mononylon (Prestes \& Landim-Alvarenga, 2017).

O manejo pós-operatório consiste na observação do animal operado até que ele seja completamente recuperado da anestesia, ser monitorado quanto ao apetite, ingestão de água, comportamento geral, na qual a condição do paciente garanta que o nível de dor do animal seja gerenciado com auxílio de analgésicos, anti-inflamatórios, antibióticos e outros agentes (PRI, 2010; Rowsell, 1991).

Como protocólo terapêutico no pós-operatório, executa-se o meloxicam associado ao cloridrato de tramadol durante três vezes ao dia, por cinco dias para analgesia, na qual a avaliação constante do bemestar deve ser conduzida visando adaptar a terapia antálgica (Mathews et al., 2014; Prestes \& LandimAlvarenga, 2017). A terapia antimicrobiana pode ser administrada com dose única de penicilina benzatina $20 \mathrm{UI} / \mathrm{kg}$ via intramuscular e cefalexina $20 \mathrm{mg} / \mathrm{kg}$ durante duas vezes ao dia por cinco dias com iogurte para melhorar a palatabilidade (Mathews et al., 2014; Prestes \& Landim-Alvarenga, 2017).

O procedimento cirúrgico é relativamente simples e não oferece um grande desafio na exposição do útero (Mathews et al., 2014; Prestes \& Landim-Alvarenga, 2017). Mas, deve-se tomar cuidado com as complicações pós-parto como, prolapso uterino, retenção de placenta, anemia, mastite, endometriose e neoplasias como leiomiomas uterinos (Courtney, 2012).

Os auxiliares cirúrgicos são responsáveis pelos neonatos, onde vão realizar os primeiros cuidados como sucção das narinas, para retirada dos fluidos que possam está obstruindo as vias aéreas, esfregar com compressa para estimular a respiração e mantê-los aquecidos (Fossum, 2014).

E para os cuidados com os neonatos, um sistema de escore Apgar criada por uma médica norte americana na qual é um método fácil e confiável, foi modificado para avaliar a vitalidade de neonatos animais. Avaliando cinco sinais facilmente verificados como a frequência cardíaca, esforço respiratório, 
irritabilidade reflexa e motilidade e coloração das mucosas, podendo assim ser utilizados na rotina clínica, evitando-se alta taxa de mortalidade (Leite et al., 2019; Vassalo et al., 2005; Veronesi et al., $\underline{2009)}$.

\section{Considerações finais}

A metodologia de pelvimetria radiográfica é de baixo custo e grande relevância para a geração dos primatas que apresentam dificuldades no momento do parto, utilizada com finalidade de informar se está apta ou não à reprodução fisiológica, ou se é necessária a intervenção cirúrgica. A cesariana indicada como método de tratamento é um procedimento simples, embora essencial os cuidados necessários com a assepsia, neonatos e as complicações pós-parto. Esse manejo reprodutivo é perceptível para a diminuição da taxa de mortalidade, evitando a extinção dos mesmos. Assim, uma conservação bem efetuada assegura populações de espécies em habitats naturais a longo prazo.

\section{Referências}

Araújo, N. L. S. (2012). Reprodução de primatas neotropicais: avanços e perspectivas. Universidade Federal de Campina Grande.

Auricchio, P. (1995). Primatas do Brasil. Terra Brasilis Editora Ltda., Arujá, São Paulo, Brasil. (Vol. 136). Terra Brasilis Editora Ltda.

Bojrab, M. J. (2005). Técnicas atuais em cirurgia de pequenos animais. Editora Roca.

Bressan, P. M., Kierulff, M. C. M., \& Sugieda, A. M. (2009). Fauna ameaçada de extinção no Estado de São Paulo (Vol. 1).

Cooper, J. E., \& Hull, G. (2017). Gorilla Pathology and Health: With a Catalogue of Preserved Materials. Academic Press.

Courtney, A. (2012). Pocket handbook of nonhuman primate clinical medicine. CRC Press. https://doi.org/10.1201/b12934.

Couto-Santos, F. R., Mourthé, Í. M. C., \& Maia-Barbosa, P. M. (2004). Levantamento preliminar da concepção de jovens estudantes sobre a conservação de primatas da Mata Atlântica em duas instituições não-formais de ensino. Ensaio Pesquisa Em Educação Em Ciências, 6(2), 151-160.

Dyce, K. M., Wensing, C. J. G., \& Sack, W. O. (2004). Tratado de anatomia veterinária. Elsevier Brasil.

Fantoni, D. T., \& Cortopassi, S. R. G. (2009). Anestesia em cães e gatos. Roca.

Ferrari, S. F., \& Rímoli, J. (1985). A Primatologia no Brasil. Revista Da Sociedade Brasileira de Medicina Tropical, 18(3), 202. https://doi.org/10.1590/s0037-86821985000300013.

Fleagle, J. G. (2013). Primate adaptation and evolution. Academic press.

Fortes, V. B., \& Bicca-Marques, J. C. (2005). Ecologia e comportamento de primatas: métodos de estudo de campo. Caderno La Salle XI, 2(207-218).

Fossum, T. W. (2014). Cirurgia de pequenos animais (4th ed., Vol. 1). Elsevier Brasil.

Garcia, L. C. F., \& Bernal, F. E. M. (2015). Enriquecimento ambiental e bem-estar de animais de zoológicos. Ciência Animal, 25(1), 46-52.

Klaumann, P. R., \& Otero, P. E. (2013). Anestesia locorregional em pequenos animais. Roca.

Landim-Alvarenga, F. C., Prestes, N. C., \& Santos, T. C. M. (2006). Manejo do neonato. Obstetrícia veterinária. Guanabara Koogan.

Lavor, M. S. L., Pompermayer, L. G., Nishiyama, S. M., Duarte, T. S., Filgueiras, R. R., \& Odenthal, M. E. (2004). Efeitos fetais e maternos do propofol, etomidato, tiopental e anestesia epidural, em cesariana eletivas de cadelas. Ciência Rural, 34(6), 1833-1839. https://doi.org/10.1590/s010384782004000600026.

Leite, A. G. P. M., Cordeiro, M. L. L., Albuquerque, S. C. M., Dias, F. E. F., Arrivabene, M., \& Cavalcante, T. V. (2019). Escore APGAR: Aplicação em neonatos caninos nascidos de parto normal e cesariana. PUBVET, 14(4), 1-5.

Lúcio, C. F. (2008). Influência das condições obstétricas ao nascimento sobre padrões de vitalidade e bioquímica neonatal na espécie canina. Universidade de São Paulo. 
Machado, A. B. M., Drummond, G. M., \& Paglia, A. P. (2008). Livro vermelho da fauna brasileira ameaçada de extinção. In Livro vermelho da fauna brasileira ameaçada de extinção (p. 1420).

Mathews, K., Kronen, P. W., Lascelles, D., Nolan, A., Robertson, S., Steagall, P. V. M., Wright, B., \& Yamashita, K. (2014). Guidelines for recognition, assessment and treatment of pain: WSAVA Global Pain Council members and co-authors of this document. Journal of Small Animal Practice, 55(6), E10-E68.

Moore, K. L. (2013). Anatomia orientada para a clínica. In Anatomia orientada para a clínica. Guanabara - Koogan.

Mosdow, G. (1976). Caesarean section in the Java monkey (Macaca irus) - a case report. Journal of Small Animal Practice, 17(8), 519-525.

Nelson, R. W., \& Couto, C. G. (2015). Medicina interna de pequenos animais (Issue 1). Elsevier Editora.

Niebauer, G. (1993). Técnicas atuais em cirurgia de pequenos animais. In M. J. Bojrab (Ed.), Current Techniques in Small Animal Surgery. Lea and Febiger.

Pafaro, V., Isola, J. G. M. P., \& Canola, J. C. (2010). A importância da pelvimetria radiografica para a reprodução de animais de companhia e de produção: Revisão de literatura. PUBVET, 4, Art-865.

Presgrave, O. A. F. (2002). Alternativas para animais de laboratório: do animal ao computador. In A. Andrade, S. C. Pinto, \& R. S. Oliveira (Eds.), Animais de laboratório: criação e experimentação. Edotora Fiocruz.

Prestes, N C, \& Landim-Alvarenga, F. C. (2017). Obstetrícia veterinária. Guanabara Koogan.

Prestes, Nereu Carlos, Ferreira, J. C. P., Ferraz, M. C., Garofalo, N. A., Simões, C. R. B., Sabino, F. A., Leal, L. S., \& Oba, E. (2014). Cesarean sections in marmosets: white-tufted marmoset (Callithrix jacchus). Veterinária e Zootecnia, 92-97.

PRI. Guidelines for Care and Use of Nonhuman Primates. Primate Research Institute, Kyoto University. Japão, p.35-37, 2010.

Rodrigues, R. C. (2010). Ciclo reprodutivo de macacos-prego (Cebus libidinosus) em cativeiro: Aspectos comportamentais e hormonais. Universidade de Brasília.

Rowsell, H. C. (1991). The Canadian Council on Animal Care--its guidelines and policy directives: the veterinarian's responsibility. Canadian Journal of Veterinary Research, 55(3), 205.

Simas, R. C., Bacchiega, T. S., Dias, L. G. G. G., Pereira, D. M., \& Dias, F. G. G. (2012). Técnica cirúrgica para cesarianas em cadelas e gatas. Revista Científica Eletrônica de Medicina Veterinária, 9(18), 1-6. https://doi.org/16797353.

Souza, I. V. de. (2010). Aspectos morfológicos do útero de Macaco Rhesus (Macaca mulattaZimmermann, 1780) em fêmeas nulíparas, primíparas e pluríparas. Universidade de São Paulo.

Stockinger, D. E., Torrence, A. E., Hukkanen, R. R., Vogel, K. W., Hotchkiss, C. E., \& Ha, J. C. (2011). Risk factors for dystocia in pigtailed macaques (Macaca nemestrina). Comparative Medicine, 61(2), $170-175$.

Teixeira, D. G. (2005). Estudo anatômico descritivo dos órgãos genitais masculinos do macaco-prego (Cebus apella Linnaeus, 1758). Universidade de São Paulo.

Valle, C. M. del R., Valle, R. del R., Monteiro, F. O. B., Castro, P. H. G., Valentim, R., Carvalho, R. de A., Muniz, J. A. P. C., \& Bombonato, P. P. (2006). Pelvimetria em macacos-da-noite (Aotus azarai infulatus-KUHL, 1820). 43(3), 370-378.

Vassalo, F. G., Silva, L. P. da, Lourenço, M. L. G., \& Chiacchio, S. B. (2005). Apgar, V. A proposal for a new method of evaluation of the newborn infant. Revista Brasileira de Reprodução Animal, Currents R, 260-267.

Veiga, G. A. L., Silva, L. C. G., Lúcio, C. de F., Rodrigues, J. A., \& Vannucchi, C. I. (2009). Endocrinologia da gestação e parto em cadelas. Revista Brasileira de Reprodução Animal, 33(1), 310.

Veronesi, M. C., Panzani, S., Faustini, M., \& Rota, A. (2009). An Apgar scoring system for routine assessment of newborn puppy viability and short-term survival prognosis. Theriogenology, 72(3), 
401-407.

Vidal, M. D., Rohe, F., Buss, G., Silva Jr, J. S., Jerusalinsky, L., Fialho, M. S., Rossato, R. S., Azevedo, R. B., Sampaio, R., \& Alves, S. L. (2012). Protocolo para coleta de dados sobre primatas em Unidades de Conservação da Amazônia. Brasília: ICMBio, 37, 7-8.

Williams, C. V, Junge, R., \& Randall, E. (2014). Prosimians. In G. West, G. Heard, \& N. Caulkett (Eds.), Zoo animal and wildlife imomobilization and anesthesia (pp. 551-559). Wiley Blackwell.

Wolfe-Coote, S. (2005). The laboratory primate. Academic Press. https://doi.org/10.1016/B978-0-12080261-6.X5000-4.

\section{Histórico do artigo:}

Recebido: 27 de maio de 2020

Aprovado: 22 de julho de 2020.

Licenciamento: Este artigo é publicado na modalidade Acesso Aberto sob a licença Creative Commons Atribuição 4.0 (CC-BY 4.0), a qual permite uso irrestrito, distribuição, reprodução em qualquer meio,

Disponível online: 4 de dezembro de 2020. desde que o autor e a fonte sejam devidamente creditados. 